\title{
Signalling blocklayout and strategy of train operation for saving energy in mass rapid transit systems
}

\author{
B.R. Ke and N. Chen
}

\begin{abstract}
An approach for assisting the design of fixed-block signalling system of mass rapid transit (MRT) systems by optimising the block layout and running speed code of each signalling block between any two neighbouring stations is presented. The object is to achieve minimum energy consumption with maximum train capacity. The apporach preadjusts the running speed restriction of trains to avoid overspeeding on an alignment. At the same time it can also shave-off unnecessary speed changes to improve passenger comfort. The concept of equiblock signalling is used to assist this research. Considering the effect of gradients and the limits of minimum headway of an ATO system and average train speed, a genetic algorithm is applied to determine the shortest length, speed codes and positions of signalling blocks. Single train running dynamics between stations is simulated based on the signalling system of an MRT system with various aspects, positions and numbers of signalling blocks through acceleration and jerk limits. An heuristic search is used to seek out the combination of train running speed code of each signalling block for energy saving.
\end{abstract}

\section{List of symbols}

$a_{s}$
$b$
$b_{s}$
$E$
$E_{n}$
$E_{\min }$
$f_{i}$
$F$
$g$
$g_{a v, n}$
$H$
$H_{s p e c}$
$J$
$l_{i, j+1}$
$l_{j, j+1}^{i}$
$l_{\text {remainder }}$

$L$

\section{$L_{\text {essential }}$}

normal acceleration

constant to determine reduce

degree of $\delta(t)$

normal deceleration

train energy consumption run-

ning between two stations

train energy consumption during

$n$th signalling block

lowest train energy consumption running between two stations

fitness value of $i$ th gene

tractive effort

gradient

average gradient of $n$th signalling

block

practical headway

minimum headway of specifica-

tion

jerk restriction

braking distance from speed code $V_{i}$ to $V_{i+1}$

braking distance from speed

code $V_{i}$ to $V_{i+1}$ at $i$ th gene

remainder length after deducting

essential length of signalling

blocks from distance between

two stations

distance between stations
C IEE, 2005

IEE Proceedings online no. 20045188

doi:10.1049/ip-epa:20045188

Paper first received 28th June and in revised form 14th December 2004

The authors are with the Department of Electrical Engineering, National Taiwan University of Science and Technology, Taipei, Taiwan, Republic of China

$L_{n}$
$L_{n}^{i}$

$N$
pop
$P$
$r$

$R$
$R_{\text {index }}$

$R_{\text {index_to_lower }}$

$R_{\text {index_to_upper }}$

$R_{\text {lower }}$

$R_{\text {upper }}$

$S B$

$t_{r}$

$T$

$T_{\max }$

$v$

$v_{a n}$

$v_{n}$

$V_{a v}$

$V_{a v, s p e c}$ essential braking distance between any neighbouring speed codes

length of $n$th signalling block ending position of $n$th signalling block of $i$ th gene

number of aspects

population size

tractive power

random number between -1 and 1

train running resistance

equi-speed code with identical speed code for each signalling block to be closest to limits of headway and average speed but not to exceed

search range of individual block from equispeed-code index to lower speed code

search range of individual block from equispeed-code index upper speed code

lower limit of possible search range of speed code

upper limit possible search range of speed code

number of signalling blocks response delay of ATO system evolved generation maximum evolved generation train running speed $n$th speed restriction of alignment

train running speed code of $n$th signalling block

practical average speed minimum average speed of specification 


$\begin{array}{ll}V_{i} & \text { ith speed code } \\ V_{j}^{i}, V_{j}^{i^{\prime}} & j \text { th speed code of } i \text { th gene before } \\ & \text { and after crossover is performed } \\ & \text { train weight } \\ & j \text { th chromosome of } i \text { th gene } \\ x_{j}^{i}, x_{j}^{i^{\prime}} & \text { before and after crossover and } \\ & \text { mutation operation is executed } \\ & i \text { th gene in population } \\ \bar{x}_{i} & \text { upper limit of jth chromosome } \\ x_{j}^{i} & \text { of } i \text { th gene } \\ & \text { random number with range in } \\ \alpha & (-1,1) \\ & \text { formula for producing a random } \\ & \text { number between }-1 \text { and } 1 ; \\ & \text { moreover, its value will gradu- } \\ & \text { ally be reduced when number of } \\ & \text { evolved generation increases } \\ & \text { snapshot time } \\ & j \text { th chromosome of } i \text { th gene for } \\ & \text { distributing proportion }\end{array}$

\section{Introduction}

The first task is to consider the transportation capacity that affects the specifications of a railway system. For designing a signalling system for mass rapid transit (MRT) systems, the minimum headway must be computed after the number of passengers per hour and the kinds of vehicles are determined. Moreover, for a fixed-block signalling (FBS) system, aspects and speed codes will be devised to fit in with the minimum headway and average train speed, and further, to obtain the proper positions of blocks. The final task is to perform operational tests and make any necessary adjustments.

Gill and Goodman [1] proposed a computer-based optimisation technique for designing signalling systems of MRT systems based on an equiblock principle. Using a formula for safe braking distance the essential and shortest block length is confirmed. Chang and Du [2] applied a genetic algorithm (GA) in place of conventional methods to improve an existing block design for equiblock signalling in an MRT system. Their primary objectives were to minimise the headway between two neighbouring stations as far as possible. Nevertheless, the time difference between the designated and practical minimum headway can be utilised to reduce energy consumption by selecting the proper block layout and running-speed code at each block on the journey.

How to minimise the headway has been discussed in [2], but the main object of this paper is to address the designated minimum headway for saving energy rather than simply the minimum headway. The designated minimum headway can simultaneously satisfy requirements of construction specification under normal conditions with a margin in minimum headway for service recovery. There are ways to reduce energy consumption of trains, for example, by decreasing the maximum running speed and running the train under coasting mode [3-7]; however, their object is to minimise energy consumption under normal conditions rather than during peak train capacity. In this paper the block-layout of signalling systems is designed and the running speed code of each block determined to minimise energy consumption and satisfy the limitations of minimum headway and average train speed when the MRT system operates during peak train capacity. Taking account of the effects of signalling systems and gradients, the block- layout of signalling systems consuming minimum energy can be devised using the least signalling blocks by comparing energy consumption with various numbers of block sections.

This research study presents a new approach for designing signalling systems for MRT systems while considers the effects of gradient between two neighbouring stations to save energy consumption of trains. Considering the minimum headway and average train speed, a genetic algorithm is used to determine the shortest length, speed codes and positions of signalling blocks. Furthermore, an heuristic search is used to seek out the combination of train running speed code at each signalling block for energy saving.

For safety and equipment cost, the length of signalling blocks must be greater than the safe braking distance. Safety is the principal consideration for designing a signalling system. Even under normal conditions, it must still have a sufficient braking distance. The design considerations are according to the requirements of the automatic train operation (ATO) system and safe braking distance. According to the effect of gradient and the restriction of headway the length can be regulated. First, for consistency of speed codes and simplifying the calculation of braking distances this paper creates the shortest length and speed codes of each signalling block by the principle of equiblock signalling and the GA method. Secondly, this paper regulates the running speed limit of trains in advance to avoid overspeeding on an alignment; moreover, it shaves off unnecessary speed change to improve passenger comfort. Thirdly, according to the given number of aspects and signalling blocks, the positions of signalling blocks are determined by using a GA. And then according to these results, the running speed code of signalling blocks for energy saving can be found for all possible positions of signalling blocks through an heuristic search. Comparing the energy consumption for different positions of signalling blocks, the lowest one is the solution.

\section{Mathematical modelling}

For a FBS system the alignment parameters of a railway, such as gradient and curvature, the performance parameters of vehicles, such as acceleration/deceleration, traction force, braking force and jerk, the design parameters of the signalling system, such as aspects, block positions and speed codes, as well as the operation parameters of the system such as headway, the number of passengers, and the time of station dwell, will influence the operation. It is too complicated to use formulations for calculating the dynamics of the railway system; therefore a computer is used to simulate the running result of vehicles in most of this research area. Based on a railway signalling system with different aspects and speed codes, the train dynamics of running between stations is simulated on a computer in this paper. The result simulated is to select the aspects, speed codes and block positions.

To meet the requirements of minimum headway and average train speed and to save energy consumption of trains, the paper proposes a procedure to complete the design of block-layout of FBS systems and to resolve the running speed code of each signalling block between two neighbouring stations. The flowchart of this procedure is shown as Fig. 1 and explained as follows. First, the GA is used to solve the shortest length of signalling blocks and speed codes for an equiblock signalling system. Secondly, unnecessary speed changes are avoided by adjusting line speed for passenger comfort and energy saving. Thirdly, 


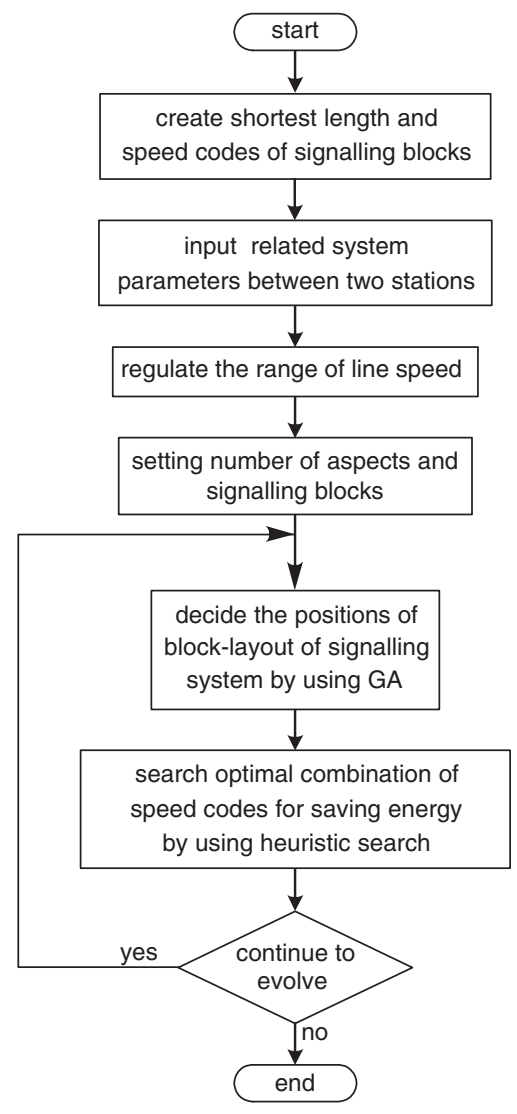

Fig. 1 Flowchart of design of block-layout and its running speed codes

after inputting the number of aspects and signalling blocks, the GA is used again to determine the positions of signalling blocks. And then the heuristic search is used to decide the optimal combination of running speed code of each signalling block for energy saving. The optimal results, including the optimal combination of running speed code and position of each signalling block, can be determined by comparing the results of evolution repeatedly. Finally, to compare the results of different numbers of aspects and signalling blocks the optimal combination of running speed code and position of each signalling block can be confirmed for the energy saving and restrictions of minimum headway and average train speed between two neighbouring stations.

Equation (1) shows the requirements of tractive effort to run at any location of a railway system. A moving train must overcome the resistance concerned with gradient, train speed and weight. The resistance of curvature and air is not large enough to affect the result for rapid transit systems. This paper adopts the formula of the Taipei Rapid Transit System. Regarding the power cost, the peak train power is considered during optimisation, but its influence on the results is not obvious. The main factors affecting peak train power are the alignment, weight and acceleration. In this paper the alignment and train weight one considered; also the train acceleration control is by PID control to meet the limits of train velocity, acceleration and jerk. In (2) the consumed power relates to the train speed as well; (3) shows that all energy consumed during a journey is the sum of all the products of snapshot time and its consumed power.

$$
\begin{gathered}
F(g, v)=a_{s}, W+R(g, v) \\
P(g, v)=F(g, v) v \\
E(g, v)=\sum P(g, v) \Delta t
\end{gathered}
$$

The object of this paper is to minimise the energy consumption for FBS transit railway systems. The major factors affecting energy consumption are the alignment gradient and train running speed; therefore selecting different signalling block-layouts and running speed codes will reach a different result. But the requirements of minimum headway and average train speed are influenced by the signalling block-layout and running speed code. The headway will drop when the train running speed increases, but the consumed energy and average speed will increase. In addition, the signalling block-layout also affects them. Therefore the objective function and constraint conditions can be shown as follows:

objective function

$$
E_{\min }=\min \sum_{n=1}^{N} E_{n}\left(L_{n}, g_{a v, n}, v_{n}\right)
$$

constraint conditions

$$
\begin{gathered}
H \leq H_{\text {spec }} \\
V_{a v} \geq V_{a v, s p e c}
\end{gathered}
$$

According to the construction specifications of the Taipei Mass Transit System, $H_{\text {spec }}$ and $V_{a v, s p e c}$ are two min and $37 \mathrm{~km} / \mathrm{h}$, respectively. The design procedure is discussed in the following Sections.

\section{Calculating shortest length of signalling blocks and speed codes in FBS systems}

The concepts of equiblock signalling of an FBS system means that they have the same braking distance between two neighbouring speed codes. Its advantage is to reduce the headway to a minimum. However, if all the signalling blocks were designed to have the same shortest length, the equipment cost would be too much; therefore the method cannot be adopted. This paper determines the block length by using the concept of equiblock signalling system and GA. The remainder of deducting the product of the number of blocks and the shortest block length from the distance between two stations is distributed to each block using GA. Consequently the shortest block length and speed codes are created.

Gill and Goodman [1] incorporate the response delay of an ATO system, the jerk limit of the train and the constant acceleration during the ATO-system response delay to formulate the braking distance between two speed codes. This paper uses this formula and GA to create the shortest block length and speed codes through an individual program. The minimum block length is shown by the following formula [1]:

$$
\begin{aligned}
I_{i, j+1}= & V_{i}\left(t_{r}+\frac{a_{s}}{J}+\frac{b_{s}}{2 J}+\frac{a_{s} t_{r}}{b_{s}}+\frac{a_{s}^{2}}{2 b_{s} J}\right)+V_{i+1}\left(\frac{b_{s}}{2 J}\right)+\frac{1}{2 b_{s}} \\
& \left(V_{i}^{2}-V_{i+1}^{2}\right)+\frac{1}{2} a_{s} t_{r}^{2}+\frac{a_{s} t_{r}}{J}+\frac{1}{3} \frac{a_{s}^{3}}{J^{2}} \\
& +\frac{1}{2} \frac{a_{s}^{2} b_{s}}{J^{2}}+\frac{a_{s} b_{s} t_{r}}{J}
\end{aligned}
$$

Genetic algorithms differ greatly from the traditional approach [8]. For example, genetic algorithms consider many points in the searching space at one time; therefore they have a better chance of obtaining a global optimal value, and of avoiding sinking into local optimal values. Genetic algorithms are suitable for any kind of objective function, and do not have complex mathematical require- 


\begin{tabular}{|c|c|c|c|c|c|c|c|c|}
\hline \multirow{2}{*}{$\begin{array}{l}\text { No. Of } \\
\text { aspects }\end{array}$} & \multicolumn{7}{|c|}{ Speed codes $(\mathrm{km} / \mathrm{h})$} & \multirow{2}{*}{$\begin{array}{l}\text { Braking } \\
\text { distances }(\mathrm{m})\end{array}$} \\
\hline & 1 & 2 & 3 & 4 & 5 & 6 & 7 & \\
\hline 4 & 80.00 & 68.88 & 52.04 & 0.00 & $\times$ & $\times$ & $\times$ & 165.66 \\
\hline 5 & 80.00 & 72.82 & 62.96 & 47.81 & 0.00 & $\times$ & $\times$ & 144.66 \\
\hline 6 & 80.00 & 75.01 & 68.44 & 59.34 & 45.23 & 0.00 & $x$ & 132.50 \\
\hline 7 & 80.00 & 76.37 & 71.71 & 65.55 & 56.95 & 43.52 & 0.00 & 124.74 \\
\hline
\end{tabular}

System parameters: maximum line speed $80 \mathrm{~km} / \mathrm{h}$; constant acceleration/decleration $+/-1.0 \mathrm{~m} / \mathrm{s}^{2}$; jerk limit $+/-1.0 \mathrm{~m} / \mathrm{s}^{3}$; response delay of ATO system $1.0 \mathrm{~s}$

ments. They use probability to find the direction of search, and hence are suitable for all kinds of optimisation problems.

Equations (8) and (9) are expressions of objective function and constraint condition for creating the shortest block length and its set of speed codes by means of GA; (8) is the objective requirement for finding a set of speed codes to meet the requirement of the shortest braking distance, while (9) is the constraint condition for ensuring that speed codes $\left\{V_{1}, V_{2}, \ldots, V_{N}\right\}$ are in descending order.

objective requirement

$$
l_{1,2}=l_{2,3}=\cdots=l_{N-1, N}
$$

Constraint condition

$$
V_{1}>V_{2}>\cdots>V_{N}
$$

The steps of this process to achieve the objective requirement using GA are explained as follows.

\subsection{Representation}

The representation is performed in real numbers because there are too many figures for a binary encoding. The advantages of real-number encoding are to save the calculation time of the computer, to reduce the error of transformation, and to increase the precision of speed codes.

\subsection{Initialising population}

The population is the space of possible solutions for searching speed codes of FBS based on equiblock principle with different number of aspects and maximum running speed. Genes in the population indicate candidate combinations of speed codes. In a gene each chromosome that is produced by random number generator to create an initial population exhibits a speed code; therefore its number will increase or decrease depending on the number of aspects.

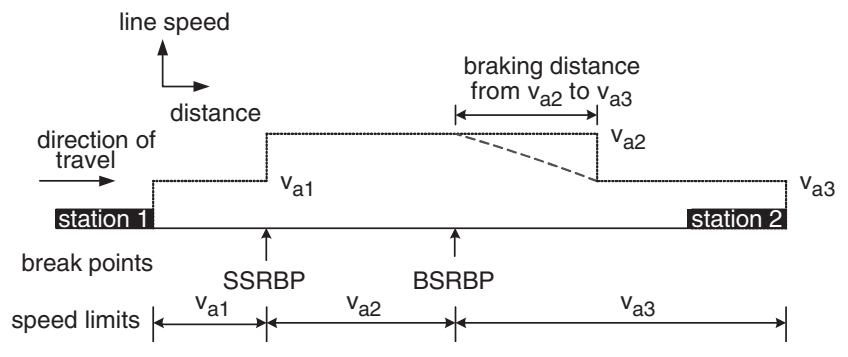

Fig. 2 Determining BSRBP and SSRBP

Notice should be taken of the order of speed codes with the constraint condition of (9); if the contents of chromosomes show that speed codes are out of descending order, they would be cancelled and reproduced. An $N$-aspect system that produces $k$ genes can be described as follows:

$$
\begin{aligned}
& \bar{x}_{1}=\left[x_{1}^{1}, x_{2}^{1}, \cdots, x_{N}^{1}\right]=\left[V_{1}^{1}, V_{2}^{1}, \cdots, V_{N}^{1}\right] \\
& \bar{x}_{1}=\left[x_{1}^{2}, x_{2}^{2}, \ldots, x_{N}^{2}\right]=\left[V_{1}^{2}, V_{2}^{2}, \cdots V_{N}^{2}\right] \\
& \vdots \\
& \bar{x}_{p o p}=\left[x_{1}^{p o p}, x_{2}^{p o p}, \cdots, x_{N}^{p o p}\right]=\left[V_{1}^{p o p}, V_{2}^{p o p}, \cdots, V_{N}^{p o p}\right]
\end{aligned}
$$

The sequence of genes will be permuted in accordance with their fitness values after creating initial population. The object is to reach the same braking distance between neighbouring speed codes. The fitness of the $k$ th gene with $N$-aspects is given by

$$
f_{k}=\sqrt{\sum_{i=1}^{N-1} \sum_{j=i+1}^{N-1}\left(l_{i, j+1}^{k}-l_{j, j+1}^{k}\right)^{2}}
$$

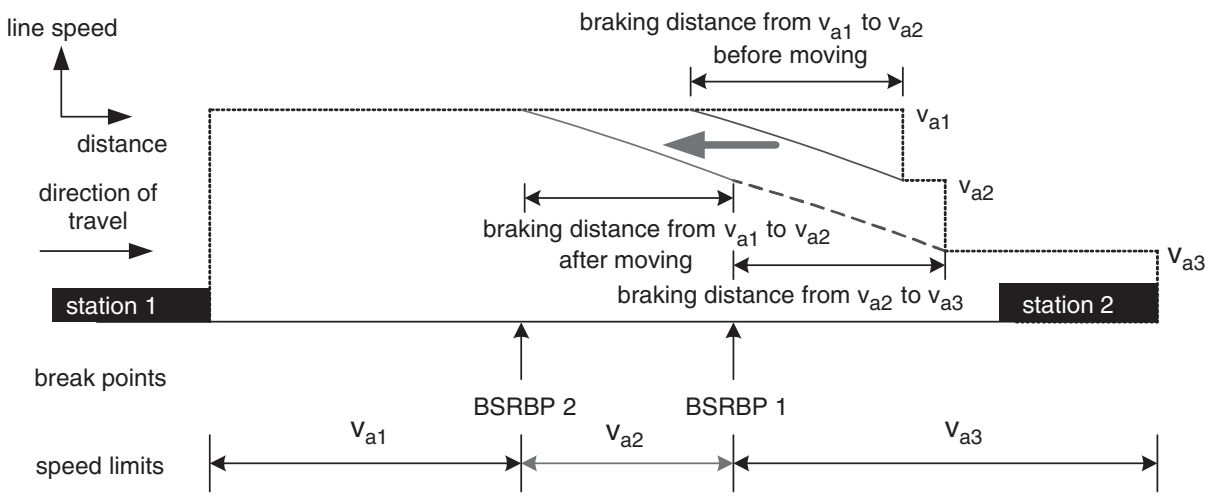

Fig. 3 Solving braking distance overlaps between two BSRBPS 


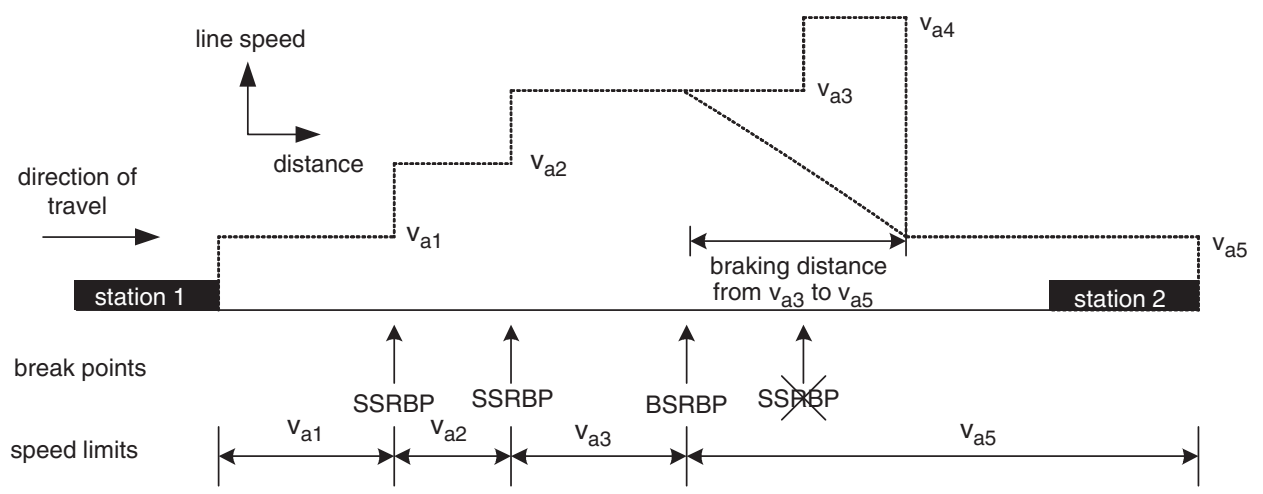

Fig. 4 Solving braking distance of BSRBP and position of SSRBP overlap
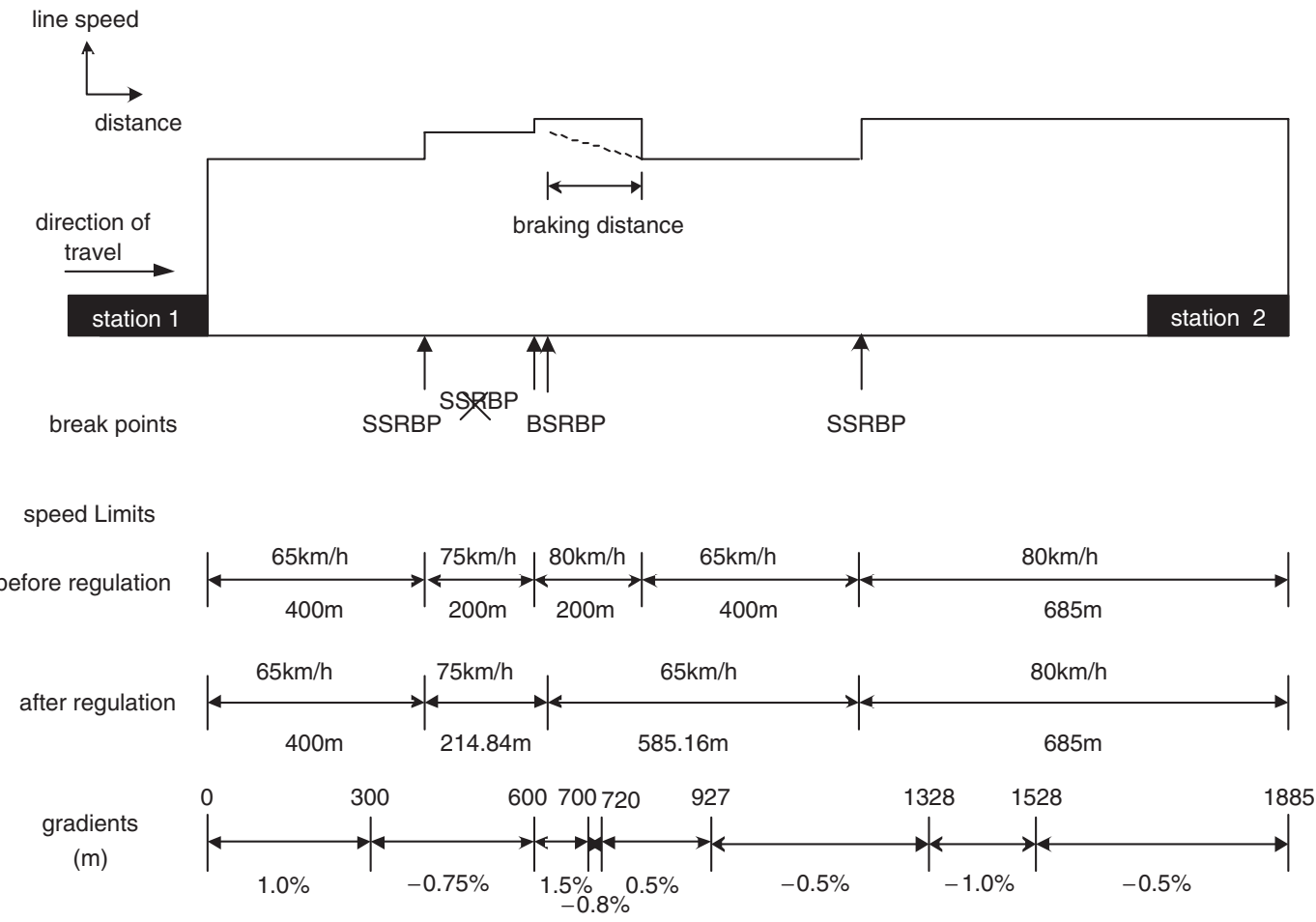

Fig. 5 Comparison of pre-and post-regulated speed restrictions of alignment

\subsection{Crossover operation}

The object of crossover operation is to produce new genes with different chromosomes. A gene shows a combination of speed codes and chromosomes reveal speed codes among a gene. New genes will become a possible solution to satisfy all requirements. The candidate genes for performing the crossover operation in the population are randomly determined. First, a random number produced between zero and one by a random number generator is assigned to each gene and compared with the crossover probability. Secondly, the crossover operation of a gene is performed when the given random number is greater than crossover probability. All genes that conformed to this condition must be picked from the population to perform the crossover with each other. Finally, the execution of the crossover operation is aimed at the individual chromosome at the same position in genes. The calculation is based on a direction-based operation [8] and its formula is given by (12). The $j$ th chromosomes $\left(x_{j}^{i^{\prime}}\right.$ and $\left.x_{j}^{i+1^{\prime}}\right)$ of new $i$ th and $(i+1)$ th genes are calculated by the sum of themselves $\left(x_{j}^{i^{\prime}}\right.$ and $x_{j}^{i+1^{\prime}}$ ) and the product of the difference between $x_{j}^{i}$ and $x_{j}^{i+1}$ and a random number $\alpha$. Because the range of random number $\alpha$ is between -1 and 1 , it is possible that new chromosomes are greater or smaller than the original chromosomes after executing this operation. The result will become a part of offspring

$$
\left\{\begin{array}{l}
x_{j}^{i^{\prime}}=x_{j}^{i}+a\left(x^{i}-x_{J}^{i+1}\right) \\
x_{j}^{i+1^{\prime}}=x_{j}^{i+1}+\alpha\left(x_{j}^{j+1}-x_{j}^{i}\right)
\end{array}\right.
$$

\subsection{Mutation operation}

The process of mutation operation is similar to the crossover operation. A random number between zero and one is assigned to each gene and its chromosomes in the population, and compared with the mutation probability. The mutation operation of a chromosome is performed when the given random numbers for the chromosome and its gene are simultaneously greater than the mutation probability. The calculation formula is given as (13) and (14) [18]:

$$
\begin{gathered}
x_{j}^{i^{\prime}}=x_{j}^{\prime}+\left(x_{j}^{\prime} U-x_{j}^{\prime}\right) \delta(T) \\
\delta(T)=r\left(1-\frac{T}{T_{\max }}\right)^{b}
\end{gathered}
$$


Table 2: List of speed limit sections before and after regulation

\begin{tabular}{|c|c|c|c|c|c|c|c|c|c|}
\hline \multicolumn{5}{|c|}{ Before regulation } & \multicolumn{5}{|c|}{ After regulation } \\
\hline No & $\begin{array}{l}\text { Start } \\
\text { point }(\mathrm{m})\end{array}$ & $\begin{array}{l}\text { End point } \\
\text { (m) }\end{array}$ & $\begin{array}{l}\text { Speed } \\
\text { limit }(\mathrm{km} / \\
\text { h) }\end{array}$ & $\begin{array}{l}\text { Length } \\
\text { (m) }\end{array}$ & No & $\begin{array}{l}\text { Start } \\
\text { point }(\mathrm{m})\end{array}$ & $\begin{array}{l}\text { End point } \\
\text { (m) }\end{array}$ & $\begin{array}{l}\text { Speed } \\
\text { limit }(\mathrm{km} / \\
\mathrm{h})\end{array}$ & $\begin{array}{l}\text { Length } \\
(\mathrm{m})\end{array}$ \\
\hline 1 & 0 & 400 & 65 & 400 & 1 & 0 & 400 & 65 & 400 \\
\hline 2 & 400 & 600 & 75 & 200 & 2 & 400 & 614.84 & 75 & 214.84 \\
\hline 4 & 800 & 1200 & 65 & 400 & 4 & 1200 & 1885 & 80 & 658 \\
\hline 5 & 1200 & 1885 & 80 & 685 & & & & & \\
\hline
\end{tabular}

The result of mutation operation for the $j$ th chromosome $x_{j}^{i}$ of $i$ th gene are calculated by the sum of itself $x_{j}^{i}$ and the product of the difference between $x_{j}^{i}$ and $x_{j}^{i}$ and $\delta(T)$. The range of $\delta(T)$ gradually reduces as the evolved generation increases and the reduced degree depends on the constant $b$. In other words, the range of mutation operation gradually reduces to speed up convergence to a better solution when approaching the end of evolution. For conforming to the descending order of chromosomes in a gene, the upper limit $x_{j}^{i^{U}}$ of a chromosome $x_{j}^{i}$ (speed code $V_{j}^{i}$ ) must be equal to its previous chromosome $x_{j-1}^{i}$ (speed code $V_{j-1}^{i}$ ). Because the range of possible optimal values is extremely concentrated in terms of the nature of objective requirements, it is not easy to escape from the local optimal value when it sinks. The method for solving this problem is to reduce the range of the mutation operation to increase both the possibilities of escaping form the local optimum value and entering the global optimum value; therefore the number of times convergence to different local optimal solutions will be possibly more than once.

\subsection{Selection operation}

When the selection operation is executed the enlarged population will include genes and the offspring produced by the crossover and mutation operations. Moreover, the genes of the offspring will be inserted into the original population in descending order of fitness. The genes of the enlarged population over the number of original population will be cancelled and the previous others will constitute a new population.

\subsection{Ending evolution}

The evolution terminates when the fitness of an optimal result is small enough or the maximum time arrives.

Table 1 shows the shortest braking distance and its set of speed codes obtained by using GA with differenct aspects.

\section{Adjustment of speed limits on alignment}

Too many changes of speed limits on an alignment will certainly cause difficulties in the planning and design of signalling systems. The approach to set speed restriction break points (SRBP) in this paper preadjusts the running speed of trains to avoid overspeeding on an alignment; moreover, it cancels unnecessary speed changes to improve passenger comfort and save energy. There are two kinds of SRBP: braking (BSRBP) and speed-raising (SSRBP). The BSRBP takes place when the speed restrictions of alignment fall; therefore it must consider the braking distance and other factors, such as the response delay of the ATO system and the train jerk limit. The SSRBP is situated at the border of two line-speed sections where the change of line speed is

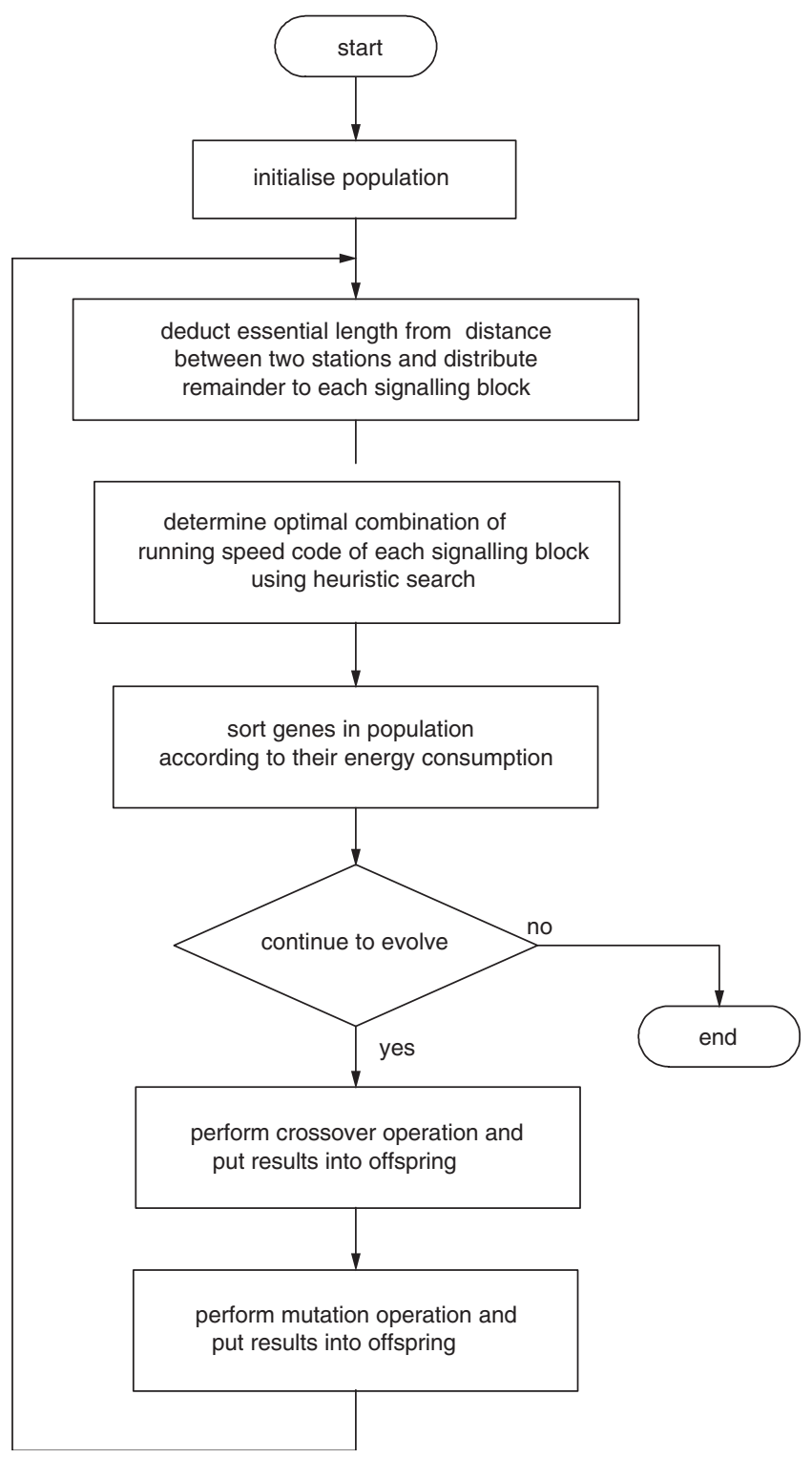

Fig. 6 Flowchart of finding optimal combination of block-layout and running speed code

from low to high. The steps for adjustment of speed restrictions on an alignment are as follows:

\subsection{Step 1: Determine BSRBP and SSRBP}

The BSRBP and SSRBP and sought from station 2 to station 1 in Fig. 2. When the speed restrictions of alignment fall, such as from $v_{a 2}$ to $v_{a 3}$, subtract the braking distance from the starting point of the lower line speed $v_{a 3}$ and settle on that point to be a BSRBP. When the speed restrictions 
of alignment rise, such as from $v_{a 1}$ to $v_{a 2}$, settle on the starting point of the higher line speed $v_{a 2}$ to be a SSRBP.

\subsection{Step 2: Check whether break points overlap between BSRBP and SSRBP or two BSRBPs from station 2 to station 1}

\subsubsection{Braking distances overlap between two} BSRBPS: In Fig. 3 the area of braking distance from $v_{a 2}$ to $v_{a 3}$ overlaps the one from $v_{a 1}$ to $v_{a 2}$. The method of solving this problem is to move the end-point of braking distance of the BSRBP 2, which is farther than station 2, to the starting point of braking distance of the BSRBP 1 , which in nearer than station 2 .

\subsubsection{Braking distance of BSRBP and posi-} tion of SSRBP overlap: In Fig. 4, within the braking distance of a BSRBP whose position is at the starting point of line speed $v_{a 5}$, a SSRBP occurs. Trains running on the railway system are unable to brake immediately to line speed $v_{a 5}$ when accelerating to $v_{a 4}$, thus the SSRBP within the section for line speed limit $v_{a 4}$, is cancelled.

\subsection{Step 3: According to method of deciding SRBP, speed restriction sections are created from station 2 to station 1}

Figure 5 compares alignment before and after regulation between station 1 and station 2 . The distance is $1885 \mathrm{~m}$ between stations. The start and end points, line speed and length of each speed restriction section are shown as Table 2. There are five speed restriction sections before regulation and four after regulation. During regulation the start point of the fourth section is moved forward for $800 \mathrm{~m}$ to $614.84 \mathrm{~m}$ because of BSRBP. The third section is too short with a length of $14.84 \mathrm{~m}$ and hence is merged into second.

\section{Exploring optimal combination of position and running speed code of each signalling block by usi- ng GA for saving energy}

After adjustment of line speed this paper explores the optimal combination of position and running speed code of each signalling block for saving energy using GA according to the various aspects and signalling blocks. Its result must meet the requirements of minimum headway and average train speed. The number of aspects and signalling blocks are related to each other. Increasing the number of signalling blocks are related to each other. Increasing the number of signalling blocks within a fixed distance, the number of aspects can be theoretically advance for reducing the headway. This advantage is not obvious when the response delay of an ATO system is taken into account, and may even have the opposite effect. At the same time the equipment cost will increase. Decreasing the number of signalling blocks can diminish the cost, but will make the headway longer. Consequently it is necessary to select a proper number of aspects and signalling blocks for saving energy and satisfying the requirement of minimum headway and average train speed.

After setting a reasonable number of aspects and signalling blocks, the product of the shortest length and the number of signalling blocks is deducted from the distance between two stations. The remainder is distributed to each signalling block by using GA for saving energy. The evolutional process using GA, including the representation, initialisation of population, crossover, mutation and selection is the same as the one in Section 1. The process whose flowchart is shown in Fig. 6 is illustrated in the following steps.

\subsection{Step 1: Content of chromosomes randomly produced to create initial population}

Once the position of platform blocks are confirmed the number of chromosomes of each gene will be less than the number of signalling blocks between the two stations by one. Genes in a population indicate different signalling block-layouts. The population with pop genes for a real number encoding in a FBS system with SB signalling blocks between two stations can be described by the following equation:

$$
\begin{aligned}
& \bar{x}_{1}=\left[x_{1}^{1}, x_{2}^{1}, \cdots, x_{S B-1}^{1}\right]=\left[\varepsilon_{1}^{1}, \varepsilon_{2}^{1}, \ldots, \varepsilon_{S B-1}^{1}\right] \\
& \bar{x}_{2}=\left[x_{1}^{2}, x_{2}^{2}, \cdots, x_{S B-1}^{2}\right]=\left[\varepsilon_{1}^{2}, \varepsilon_{2}^{2}, \cdots, \varepsilon_{S B-1}^{3}\right] \\
& \vdots \\
& \bar{x}_{p o p}=\left[x_{1}^{p o p}, x_{2}^{p o p}, \cdots, x_{S B-1}^{p o p}\right]=\left[\varepsilon_{1}^{p o p}, \varepsilon_{2}^{p o p}, \cdots, \varepsilon_{S B-1}^{p o p}\right]
\end{aligned}
$$

\subsection{Step 2: After deducting essential length of signalling blocks from distance between two stations, remainder is distributed to each signalling block}

The essential length $L_{\text {essential }}$ of each signalling block for different aspects is obtained by the braking distance between any two neighbouring speed codes in Table 1. The distribution method is according to the proportion of each random number to the sum of total random number within a gene. The distribution result of each gene according to (15) can be described as follows:

$$
\begin{gathered}
l_{\text {remainder }}=L-S B \times L_{\text {essential }} \\
L_{n}^{i}=L_{n-1}^{i}+l_{\text {remainder }} \times \frac{\varepsilon_{n}^{i}}{\sum_{j=1}^{S B-1} \varepsilon_{j}^{i}} \quad 1 \leq n \leq S B
\end{gathered}
$$

where $L_{0}=0$ and $L_{S B}=L$.

\subsection{Step 3: Using heuristic search to determine optimal combination of running speed code of signalling blocks for saving energy with different block-layouts}

The energy consumption of trains is a function of the gradient and running speed code of each signalling block. The energy consumption of a single train is defined as the fitness function of GA for achieving the goal of energy saving. Moreover, the gradient of alignment is used to assist in deciding the acceleration or deceleration of trains for reducing the search path. When a new gene is produced its optimal combination of running speed code of each signalling block is found by using the heuristic search described in Fig. 7. In other words, it is necessary to reenforce operation simulations of a single train between stations based on the same block-layout. Genes are rearranged by the results of energy consumption in descending order. The flowchart of all processes in step 3 is shown as Fig. 7 and is explained as follows:

(i) According to the descending speed codes, the operation simulations for a single train will be implemented for finding the $R_{\text {index }}$ that is an identical speed code for each signalling block to be closest but not exceed the limits. These limits include the headway and average train speed. Next, selecting the upper and lower range of speed codes to 


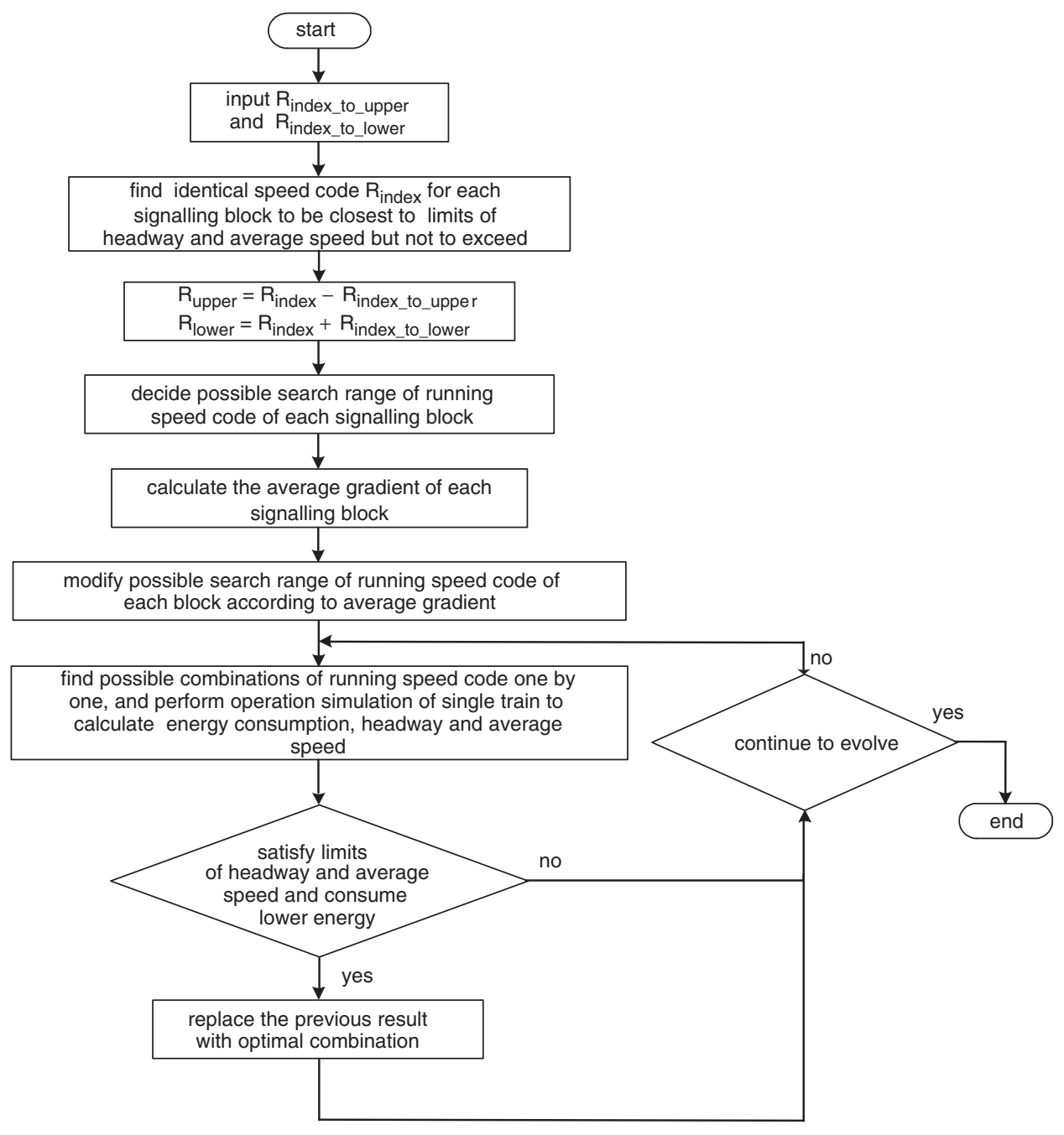

Fig. 7 Flowchart of finding optimal running speed code

be $R_{\text {index_to_upper }}$ and $R_{\text {index_to_lower }}$, reduce the search space. $R_{\text {index_to_upper }}$ and $R_{\text {index_to_lower }}$ are exploited to determine $R_{\text {upper }}$ and $R_{\text {lower }}$ shown as (18) and then improper speed codes are cancelled within each signalling block because of exceeding the line speed.

$$
\begin{aligned}
R_{\text {upper }} & =R_{\text {index }}-R_{\text {index_to_upper }} \\
R_{\text {lower }} & =R_{\text {index }}+R_{\text {index_to_lower }}
\end{aligned}
$$

(ii) Calculating the average gradient of each signalling block is to be the basis of determining the running speed code. The train decelerates or cruises for saving energy on the uphill area; therefore the search range of running speed code must be from $R_{\text {index }}$ to $R_{\text {lower }}$. The search range must be form $R_{\text {upper }}$ to $R_{\text {index }}$ in the downhill area.

(iii) The operation simulations of single train are performed for calculating the energy consumption, headway and average speed in accordance with all combinations of reasonable running speed code of each block. The optimal combination of running speed code is found for saving energy from this signalling block-layout.

To continue the example of Fig. 5, the system parameters are the same as Table 1 and the number of aspects and

\begin{tabular}{|c|c|c|c|c|c|c|c|c|c|c|}
\hline Block number & 1 & 2 & 3 & 4 & 5 & 6 & 7 & 8 & 9 & 10 \\
\hline $\begin{array}{l}\text { Content of } \\
\text { chromosomes }\end{array}$ & 0.1421 & 0.1935 & 0.1679 & 0.1346 & 0.0494 & 0.1405 & 0.0535 & 0.0316 & 0.0868 & \\
\hline Block lengths (m) & 203.2 & 224.4 & 213.9 & 200.1 & 165.0 & 202.6 & 166.7 & 157.7 & 180.4 & 171.0 \\
\hline $\begin{array}{l}\text { End point of blocks } \\
\text { (m) }\end{array}$ & 203.2 & 427.6 & 641.5 & 841.6 & 1006.6 & 1209.2 & 1375.9 & 1533.6 & 1714.0 & 1885.0 \\
\hline Speed limits $(\mathrm{km} / \mathrm{h})$ & 65 & 65 & 65 & 65 & 65 & 65 & 80 & 80 & 80 & 80 \\
\hline $\begin{array}{l}\text { Average gradients } \\
(\%)\end{array}$ & 1.0 & 0.0047 & -0.3137 & 0.6626 & 0.0176 & -0.5 & -0.6436 & -0.9823 & -0.5 & -.05 \\
\hline $\begin{array}{l}\text { Optimal combina- } \\
\text { tion of running } \\
\text { speed code }(\mathrm{km} / \mathrm{h})\end{array}$ & 47.81 & 47.81 & 62.96 & 47.81 & 47.81 & 62.96 & 62.96 & 62.96 & 62.96 & 62.96 \\
\hline
\end{tabular}

Table 3: Content of a gene in initial population and its derived data 


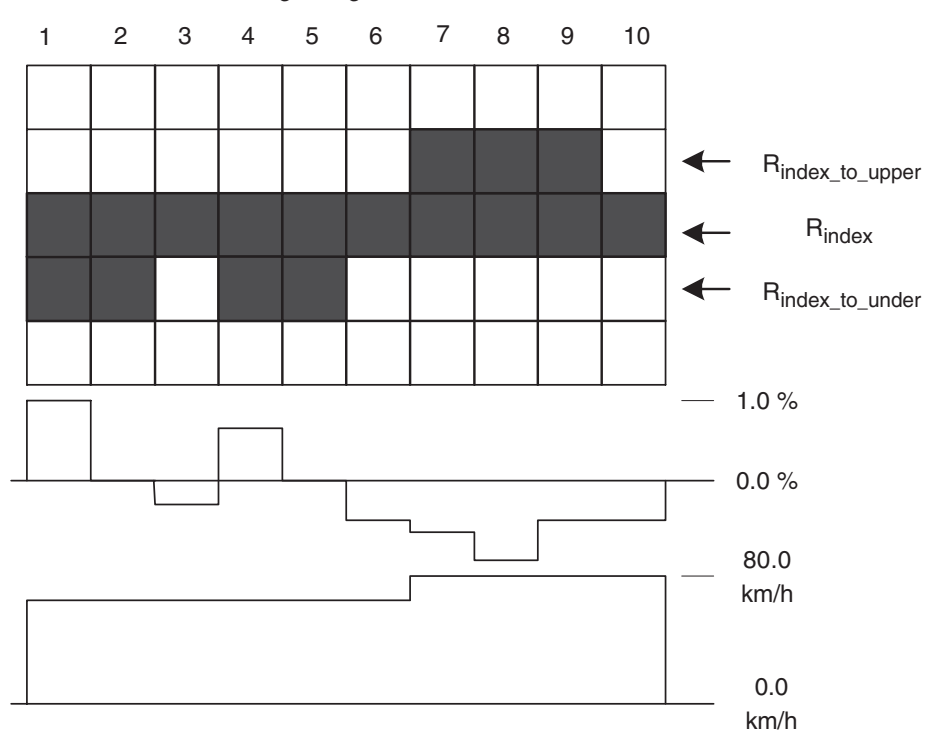

Fig. 8 Possible search range of running speed code

Table 4: Optimal results for different number of aspects and signaling blocks

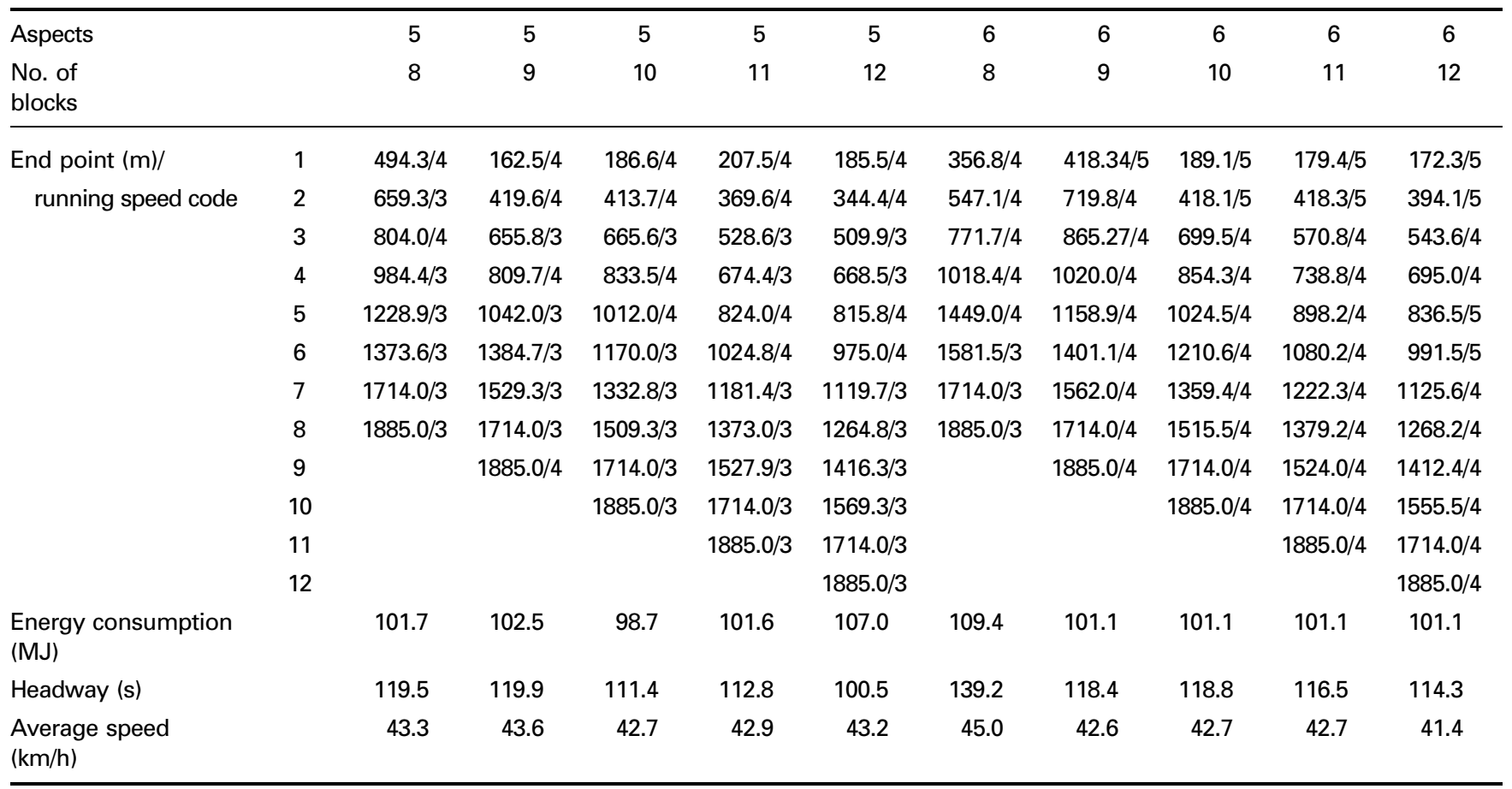

signalling blocks are set to be 5 and 10 , respectively. The dwell time is 20 seconds. The limits of headway and average train speed are 120 seconds and $40 \mathrm{~km} / \mathrm{h}$. The shortest block length and speed codes are acquired from Table $1 ; 144.66 \mathrm{~m}$ and $\{80,72.88,62.96,47.81,0 \mathrm{~km} / \mathrm{h}\}$, respectively. The content of one gene with nine chromosomes in the initial population and derived data are shown in Table 3. The data include positions, average gradients and the optimal combination of running speed code for saving energy in each signalling block. The contents of chromosomes have been normalised. The remainder, after deducting the essential length from the distance between two stations, is $442.1 \mathrm{~m}$. It is distributed to each signalling block in accordance with the proportion of the content of each chromosome to the sum of them. The length of each block is calculated from the result of distribution plus the shortest block length and is then changed into mileage. The length of the last signalling block that is named as the platform signalling block is the length of the train plus $30 \mathrm{~m}$. Signalling block 10 has a fixed length $(171 \mathrm{~m})$; therefore there are nine chromosomes in a gene. After calculating the average gradient of each block the possible search range is determined. Both $R_{\text {index_to_upper }}$ and $R_{\text {index_to_lower }}$ are assumed to be one. Because the identical 


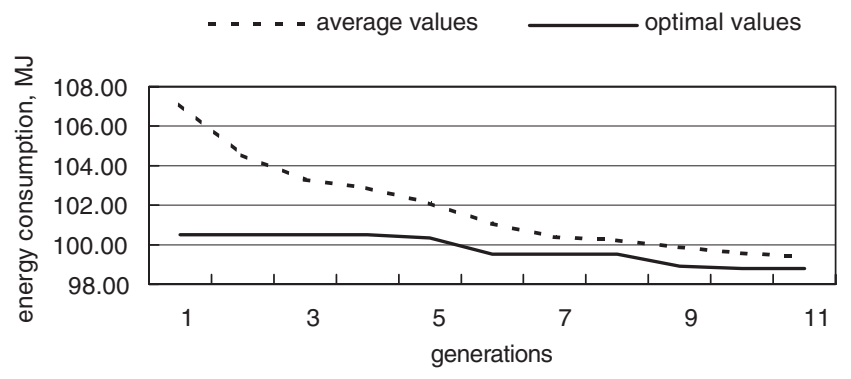

Fig. 9 Energy consumption of each generation after optimisation

speed code $R_{\text {index }}$ is searched out and its value is three, $R_{\text {upper }}$ and $R_{\text {under }}$ are calculated by (16) to be two and four, respectively. After deleting the speed codes exceeding the line speed, the possible search range of running speed code of each signalling block is shown in Fig. 8. In this Figure two line speed limits are 65 and $80 \mathrm{~km} / \mathrm{h}$, respectively. The search range of heuristic search is determined by the average gradient of each signalling block and indicated by the shadow area. Finally, the optimal combination of running speed code for saving energy is found out in this block-layout, and its headway and average train speed are $110.67 \mathrm{~s}$ and $42.502 \mathrm{~km} / \mathrm{h}$, respectively.

\subsection{Step 4: Executing crossover and mutation operations between different genes produces new offspring}

The formulations of crossover and mutation operation are the same as (12)-(14) for producing the offspring. When executing the crossover operation each gene in the population is assigned a random number to compare with a given crossover probability. If the assigned number is greater than crossover probability, the crossover operation will be executed and its results put into the offspring. Similarly, if the random assigned to each gene is greater than the mutation probability, the results of mutation operation will be put into the offspring.

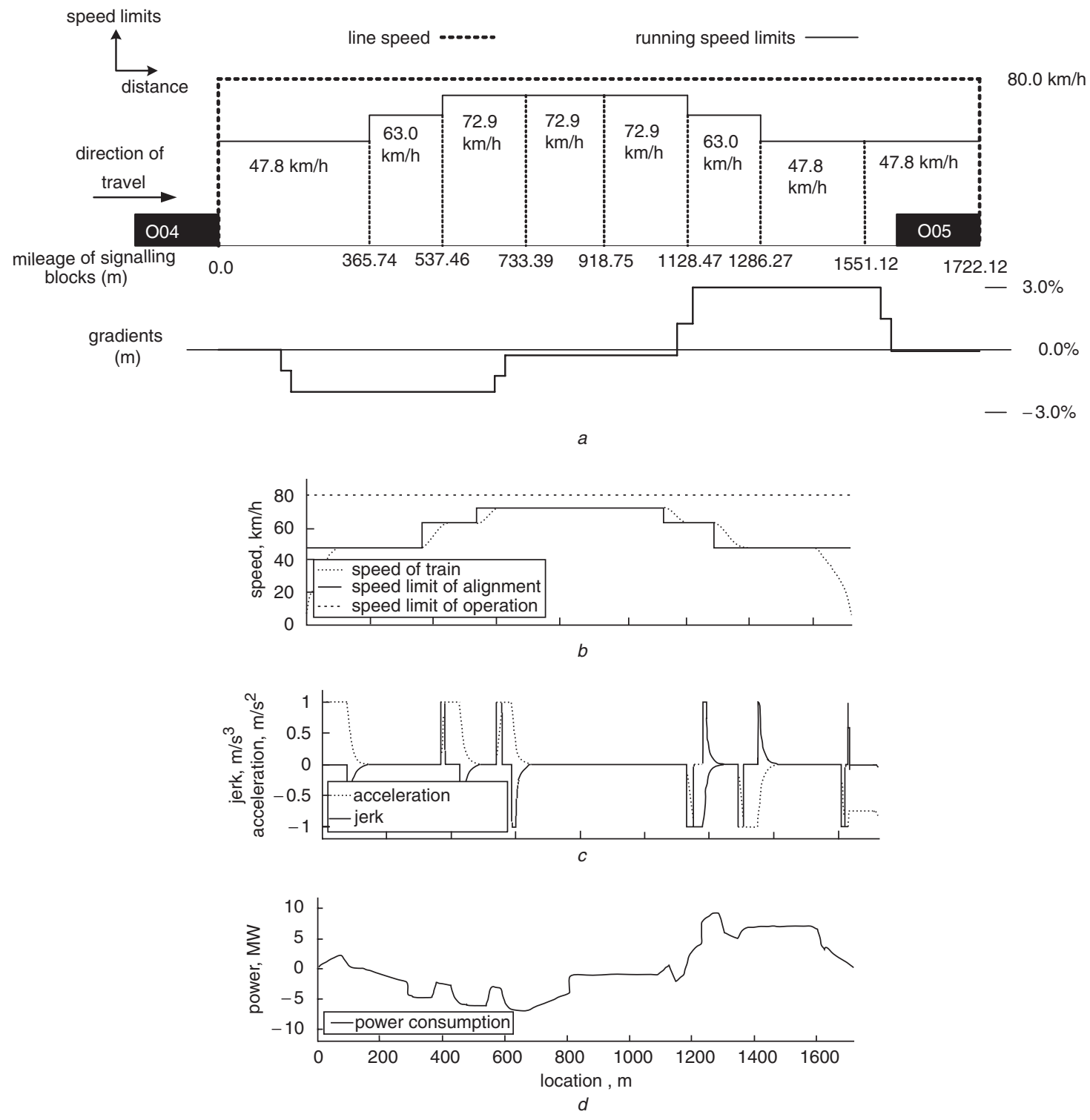

Fig. 10 Optimal result of signalling block-layout and running speed code with five aspects from stations O04 to O05 $a$ Block-layout of signalling system

$b$ Location-speed of train and -speed limit of operation and alignment curve

$c$ Location-acceleration and -jerk of train

$d$ Location-power consumption curve 


\subsection{Step 5: Previous steps are continually repeated until evolution is complete}

The work from steps 2 to 4 will be performed for the genes of an offspring. Their results will be compared with the initial population. Better ones will be reserved for a new population until the evolution is finished unless the given evolution time arrives.

Similarly, to continue the example of Fig. 5 with the same parameters, the crossover and mutation probabilities are set to be 0.8 and 0.4 , respectively. The lowest and average energy consumption for each gene in the population is shown as Fig. 9. The difference between the lowest and average values reduces gradually. This means that both of them converge to the same value. After evolving ten times, the end-points of each signalling block derived from the optimal result are $\{186.5,424.8$, $662.4,842.4,1008.8,1190.4,1371.1,1540.5,1714.0$, $1885.0 \mathrm{~m}\}$ from start station to end station. Under the limits of headway and average speed, the optimal combination of running speed code for saving energy is $\{4,4,3,4,4,3,3,3,3,3\}$. In other words, the running speed of each signalling block is $\{47.81,47.81$, $62.96,47.81,47.81,62.96,62.96,62.96,62.96,62.96 \mathrm{~km} / \mathrm{h}\}$. Its results are shown as Table 4 to determine the position and running speed code of each signalling block with different numbers of aspects and signalling blocks. It is obvious that the energy consumption for a block-layout with five aspects and ten signalling blocks is the lowest and that the requirements of headway and average train speed are satisfied.

\section{Results}

Figures 10 and 11 show the optimal result of signalling block-layout and running speed code of each signalling block between stations O04 and O05 on the Hsinchuang line of the Taipei Rapid Trasit System produced by using the proposed approach in this paper. The example is common to mass rapid transit systems. The only line speed is $80 \mathrm{~km} / \mathrm{h}$. There are downhill and uphill areas in the train starting and stopping stages, respectively. This design advantage is to assist
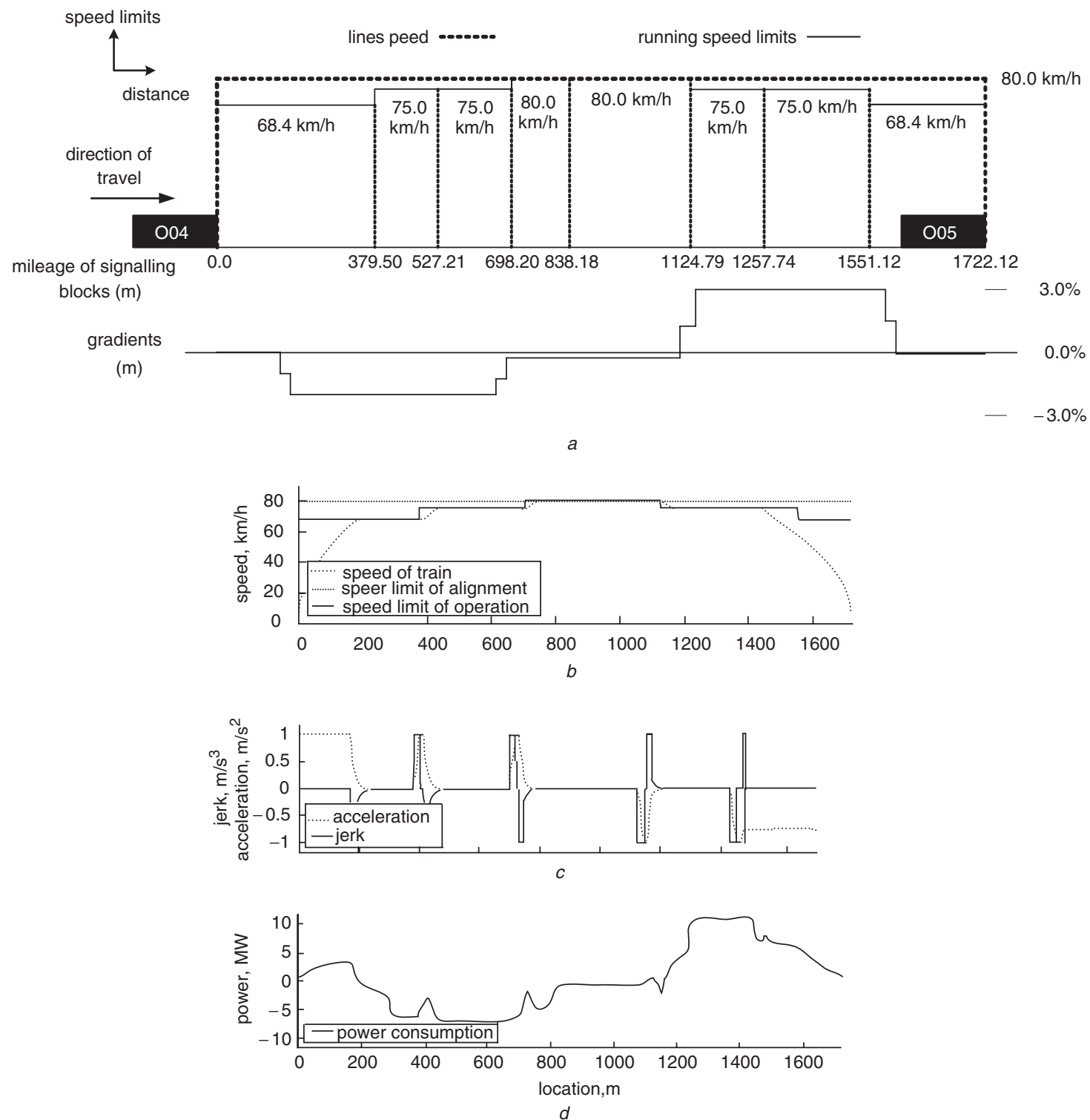

Fig. 11 Optimal result of signalling block-layout and running speed code with six aspects from stations O04 to O05 $a$ Block-layout of signalling system

$b$ Location-speed of train and -speed limit of operation and alignment curve

$c$ Location-acceleration and -jerk of train

$d$ Location-power consumption curve 
train operation in accelerating and decelerating. Fig. $10 a$ shows the optimal block-layout of five-aspect signalling system with eight signalling blocks and different running speed code on each signalling block for the lowest energy consumption. The curves of train speed and speed limit of alignment and operation are shown as Fig. $10 b$. Train acceleration is by PID control to meet the requirements of train velocity, acceleration and jerk, and their results are shown as Fig. $10 b$ and $10 d$. Figure $10 c$ shows the power consumption of a train running between stations. The headway and train average speed meet the specification requirements and are $119.51 \mathrm{~s}$ and $42.16 \mathrm{~km} / \mathrm{h}$, respectively. The optimal result of six-aspect signalling system is shown as Fig. 11 which also has eight signalling blocks. The headway and train average speed are $118.12 \mathrm{~s}$ and $47.62 \mathrm{~km} / \mathrm{h}$, respectively. To compare their energy consumption and peak power, the first is lower than the last about 3.1 and $16.05 \%$, respectively. This approach is not only common to mass rapid transit systems, but also suitable for alignments with many changes of speed limits and gradients.

\section{Conclusions}

An approach has been proposed for optimising the block-layout of FBS systems and determining the running speed cod of each signalling block between two neighbouring stations. It has emphasised minimum energy consumption for each running train and the lowest number of signalling blocks for equipment cost under the constraint of minimum headway and average train speed with train maximum capacity. Its main object is to address the designated minimum headway for saving energy rather than to obtain the practice minimum headway.

Train acceleration control is by PID control to meet the limits of train velocity, acceleration and jerk. The methods of equiblock signalling and GA are introduced to the design of signalling block-layout. The fitness of GA is determined by performing the single train movement simulation to calculate the energy consumption, headway and average train speed. Due to preadjusting the train running speed to meet the line speed and determining the running speed code by considering the average gradient of each signalling block, this approach also demonstrates its advantage when the railway system has many changes of speed limits and gradients.

A methodical approach is important for simplifying the design of block-layout of singalling systems. The computer-based technique proposed assists the design of block-layout for saving energy. It eases the design, reduces the time taken for design, and makes possible future applications. For example, the technique could be used to determine running speed codes under normal conditions and service recovery.

\section{References}

1 Gill, D.C., and Goodman, C.J.: 'Computer-based optimisation techniques for mass transit railway signalling design', IEE Proc.- B, 1992, 139, (3)

2 Chang, C.S., and Du, D.: 'Improved optimisation method using genetic algorithm for mass transit signalling block-layout design', IEE Proc.Electr. Power Appl., 1998, 145, (3)

3 Chang, C.S., and Sim, S.S.: 'Optimising train movements through coast control using genetic algorithm', IEE Proc.- Electr. Power Appl., 1997, 144, (1)

4 Hwang, H.-S.: 'Control strategy for optimal compromise between trip time and energy consumption in a high-speed railway', IEEE Trans, Syst., Man Cybern. - Part A, 1998, 28, (6)

5 Chang, C.S., Xu, D.Y., and Quek, H.B.: 'Pareto-optimal set based multiobjective tuning of fuzzy automatic train operation for mass transit system', IEE Proc.- Electr. Power Appl., 1999, 146, (5)

6 Chang, C.S., and Xu, D.Y: 'Differential evolution based tuning of fuzzy automatic train operation for mass rapid transit system', IEE Proc-- Electr. Power Appl., 2000, 147, (3)

7 Khmelnitsky, E.: 'On an Optimal control problem of train operation', IEEE Trans. Autom. Contral, 2000, 45, (7)

8 Gen, M., and Cheng, R.: 'Genetic algorithms and Engineering design' (Wiley, 1997) 